\title{
Behavioral Treatments for Non-Rapid Eye Movement Parasomnias in Children
}

\author{
Stacey L. Simon ${ }^{1}$ Kelly C. Byars ${ }^{2}$
}

Published online: 9 July 2016

(C) Springer International Publishing AG 2016

\begin{abstract}
Non-rapid eye movement (NREM) parasomnias are common in childhood and include a range of clinical presentations including confusional arousals, sleepwalking, sleep terrors, and sleep-related eating disorder. While parasomnia episodes can be dramatic and elicit concern from parents, parasomnias are predominantly benign and self-limited. A comprehensive clinical evaluation is necessary for appropriate diagnosis and ruling out underlying medical disorders. For youth with parasomnia episodes that are infrequent or limited in severity, standard treatment recommendations, including safety measures and minimizing triggering factors, are highlighted. Scheduled awakening is an evidence-based behavioral treatment that can be effective at decreasing or resolving frequent and severe NREM parasomnias but may be burdensome for parents to implement.
\end{abstract}

Keywords Pediatrics $\cdot$ Parasomnia $\cdot$ Sleepwalking $\cdot$ Sleep terrors $\cdot$ Confusional arousals $\cdot$ Sleep disorders $\cdot$ Sleep treatment $\cdot$ Children $\cdot$ Adolescents

This article is part of the Topical Collection on Behavioral Therapy

Stacey L. Simon

Stacey.Simon@childrenscolorado.org

Kelly C. Byars

Kelly.Byars@cchmc.org

1 Children's Hospital Colorado and University of Colorado Anschutz Medical Campus, 13123 East 16th Avenue, Box B395, Aurora, CO 80045, USA

2 Cincinnati Children's Hospital and University of Cincinnati College of Medicine, ML2021 3333 Burnet Avenue, Cincinnati, OH 45229-3039, USA

\section{Introduction}

Non-rapid eye movement (NREM) parasomnias are defined as disorders of arousal and encompass unwanted physical and behavioral actions or events that occur with sleep [1]. NREM parasomnias encompassed under disorders of arousal in the international classification of sleep disorders-third edition (ICSD-3 [1]) include confusional arousals, sleepwalking, sleep terrors, and sleep-related eating disorder. While distinct in their presentation, these disorders all include several key features, including (1) recurrent episodes of incomplete awakening, (2) the individual is unresponsive or inappropriately responsive during the episode, and (3) lack of recall for the episode. NREM parasomnia events typically occur during the first $1 / 3$ of the night, when deep/slow-wave sleep is most prevalent $[2,3]$. Children with parasomnias demonstrate faster alternations of amplitude of slow EEG bursts during slowwave sleep [4]. In children, these dramatic and disruptive events can be prolonged and elicit strong feelings of concern from parents and caregivers but have no negative impact on and are typically not distressing to the child $[5,6]$.

NREM parasomnias are common in young children. Prevalence of sleep terrors is $1-6 \%$ and peaks at age 1.5 years; rates of sleepwalking range from $4-40 \%$ and peak prevalence is 10 years of age $[5,7,8 \cdot]$. Confusional arousals are relatively common with rates of prevalence estimated up to $17.3 \%$ [5]. Pediatric prevalence rates for sleep-related eating disorder are not available in the published literature, and average age of onset tends to be in early adulthood (22-27 years), though one case series reported the majority of patients had onset of symptoms in adolescence $[9,10]$.

Parasomnias are predominantly benign and self-limited, often decreasing or remitting by late childhood and adolescence $[5,11]$. Predisposing factors include a family history, and precipitating factors include those which deepen sleep, 
such as young age, sleep deprivation, certain medications (e.g., neuroleptics, sedative hypnotics, stimulants, and antihistamines), and fever/illness [2, 5, 12•]. Sleep disorders such as obstructive sleep apnea (OSA), restless leg syndrome (RLS), or periodic limb movement disorder (PLMD) may mimic or trigger parasomnia episodes [13]. Parasomnias may also be triggered by sleep disruption, such as from pain, stress, environmental factors (e.g., noise, sleeping in unfamiliar setting, change in or inconsistent sleep schedule), or a full bladder. There is no evidence of an association of parasomnias with psychopathology, particularly in children $[5,14,15]$.

\section{Types of NREM Parasomnias}

\section{Sleepwalking}

While sleepwalking, by definition, is characterized by ambulation, sleepwalking episodes may begin by the child sitting up in bed, exhibiting a blank stare, and doing repetitive, automatic behaviors such as picking at clothes or bed linens before getting up and walking around [2]. Children may walk around the room, enter other rooms, or in extreme cases, even leave the house. During a sleepwalking episode, youth have decreased awareness and responsiveness to their surroundings. They are more clumsy and uncoordinated, which may result in injury, such as tripping, falling down stairs, or even walking through a window [2]. Some individuals exhibit complex behaviors during sleepwalking episodes, such as cooking, eating, or driving. Sleepwalking episodes usually last less than $15 \mathrm{~min}$, but some may be longer. Attempts by caregivers to awaken a child during a sleepwalking episode may result in agitation or aggression and typically fails to produce arousal.

\section{Sleep Terrors}

A child exhibiting a sleep terror may abruptly sit up with an expression of fear, scream, appear frightened, and be inconsolable. Sleep terrors are typically accompanied by physiological symptoms of autonomic arousal, including rapid breathing, tachycardia, sweating, dilated pupils, and increased muscle tone [5]. Children are unresponsive to their environment, and attempts by parents to console or awaken them are ineffective, seemingly ignored, and may result in disorientation and confusion. A sleep terror episode typically lasts from $30 \mathrm{~s}$ to $3 \mathrm{~min}$, and children often return to sleep afterwards with no recollection for the event the next morning.

\section{Confusional Arousals}

Confusional arousals are characterized by episodes of confused or slowed thinking, disorientation, and inappropriate responsiveness to external stimuli. Children may exhibit automatic behaviors, such as picking at clothing or bed linens, kicking or thrashing, or using objects inappropriately [2]. They may moan, cry out, or sit up, but typically do not experience significant distress or high levels of activity. These episodes are not accompanied by expressions of fear or sleepwalking. Attempts to wake or console the child often result in agitation or upset [5]. The confusional arousals typically last up to $5-15 \mathrm{~min}$, followed by lack of memory for the event [5].

\section{Sleep-Related Eating Disorder}

Sleep-related eating disorder (SRED) consists of involuntary eating or drinking during a partial arousal from sleep. The eating is typically rapid, messy, and described as "out of control" [10]. Consumption often includes foods high in carbohydrates and calories, unusual combinations of foods, and sometimes nonnutritive substances [5]. Those with SRED may become agitated or upset if the episode is interrupted, and have little or no memory for the event [9].

\section{Evaluation}

Prior to initiating treatment, a comprehensive evaluation is imperative for correct diagnosis and rule out of an underlying medical sleep disorder (e.g., OSA, RLS, PLMD), or nocturnal seizures. Assessment primarily relies on parent- or caregiverreport since, in most cases, children have no recollection for the events. Thorough inquiry regarding what events are occurring, their timing, and duration, and detailed description of the child's movements and behaviors are recommended, in addition to a comprehensive sleep history. Home videos can be helpful in addition to parents' descriptions. While unlikely to capture a parasomnia event, polysomnography may be indicated in order to rule out a medical sleep disorder, and EEG should be conducted if nocturnal seizures are suspected. Prompt treatment of any identified comorbid sleep problems is strongly recommended as a first-line intervention as it may also improve or resolve parasomnias.

\section{Treatment}

Once a diagnosis of NREM parasomnia is confirmed, treatment options can be discussed with the parent or caregiver. Of note, the existing literature on parasomnia treatment has primarily focused on sleepwalking and sleep terrors. Thus, while presentation differs for the various types of NREM parasomnias, based on the available literature, it is assumed that treatment recommendations are the same across subtypes. Collaborative discussion about the frequency and severity of the parasomnia episodes as well as parents' personal 
preferences is useful for determining which treatment methods to pursue. In many cases, NREM parasomnias will resolve spontaneously without need for intervention. In these cases and/or when the frequency and severity of parasomnia episodes are limited, standard treatment recommendations include providing education and reassurance to parents and caregivers $[3,16]$.

\section{Standard Treatment Recommendations}

Parents should be advised not to wake the child during parasomnia episodes, as it can prolong or worsen the event, and avoid discussing the episode the next day, as this may cause unnecessary anxiety or embarrassment for the child [5]. Safety measures are also critical in the overall care plan for treating NREM parasomnias. As such, safety recommendations should include the following steps, as needed: clearing the bedroom of obstructions, securing doors and windows, placing the child's mattress on the floor, having the child sleep on the first floor of the home to avoid falling down stairs, installing locks and/or alarms on windows and doors, and covering windows with heavy curtains.

Minimizing triggering factors should also be a focus of treatment. If sleep deprivation is a significant concern, an intervention targeting adequate sleep should be a priority. Parents should be provided information regarding ageappropriate sleep needs. Clinical providers should also work collaboratively with families to develop strategies to increase sleep duration, such as eliminating caffeine and ensuring enough time in bed to allow for adequate sleep opportunity. Other barriers to obtaining adequate sleep (e.g., insomnia) should be explored and addressed as needed.

\section{Scheduled Awakenings}

If a child exhibits a chronic ( $>3$ episodes per month) and severe (almost nightly, or at least multiple times per week) pattern of NREM parasomnia episodes which is significantly disruptive to the child or family, scheduled awakenings may be indicated $[11,16]$. Scheduled awakenings may be appropriate for chronic and severe parasomnias after a comprehensive evaluation has confirmed diagnosis and when episodes occur at a predictable time each night. Additionally, parents or caregivers should be fully informed about the intervention and the significant demands of the treatment protocol, and express a willingness to implement the protocol consistently over at least 1-4 weeks. Scheduled awakenings is not an appropriate intervention if the child has an underlying and untreated primary sleep disorder (e.g., OSA), episodes are infrequent, or their timing is unpredictable, if parasomnias could be more easily managed using the above described standard treatment strategies, if sleep deprivation is a significant problem and possible contributor to parasomnias (in which case increasing sleep time should be the focus of intervention), or lastly, if the parent or caregiver is unwilling or unable to implement the protocol [16].

The treatment protocol for scheduled awakenings generally involves three phases: (1) baseline monitoring and treatment planning, (2) active treatment, and (3) treatment fading and termination (see Table 1) [16].

\section{Baseline Monitoring and Treatment Planning}

Careful documentation of the timing and frequency of parasomnia episodes is essential for successfully utilizing scheduled awakenings. Monitoring of the child's sleep and documenting events on a sleep diary must be completed by the parent or caregiver. Ideally, a baseline assessment period of at least 2 weeks is recommended in order to gain an accurate picture of the child's typical sleep patterns. Objective assessment using actigraphy may also be helpful if available, but a concurrent sleep diary should still be completed by parents, as it is not possible to determine whether apparent "awakenings" during an actigraphy study are due to partial arousal episodes or true awakenings. After the data have been collected, the clinician and family should review it together. If the episodes are indeed occurring frequently and predictably, then a specific treatment plan can be developed. First, the sleep diary should be used to determine the typical time of parasomnia episode onset. Then, the sleep diary can be used to calculate the average latency from the time the child falls asleep until the onset time of partial arousal episodes. These two time points are then used to determine the pattern of onset of partial arousal episodes and to determine the optimal timing for scheduled awakenings, approximately $15-30 \mathrm{~min}$ prior to the estimated onset time of the partial arousal episode.

\section{Active Treatment}

Once the treatment plan has been developed, parents should awaken their child at the predetermined time each night. The awakenings can be accomplished with light touch or verbal prompts until the child opens his/her eyes or verbalizes he/she

Table 1 Phases of treatment for scheduled awakenings

\begin{tabular}{ll}
\hline Phase of treatment & Focus \\
\hline $\begin{array}{l}\text { 1. Baseline monitoring } \\
\text { and treatment planning }\end{array}$ & $\begin{array}{c}\text { Clinical assessment, monitoring with } \\
\text { sleep diary, calculating optimal } \\
\text { time for awakenings } \\
\text { Implement scheduled awakenings, } \\
\text { continue sleep diary monitoring, } \\
\text { adjust treatment as necessary } \\
\text { 2. Active treatment }\end{array}$ \\
$\begin{array}{l}\text { Decrease frequency of awakenings } \\
\text { based on frequency of events, } \\
\text { continue sleep diary monitoring }\end{array}$ \\
\hline
\end{tabular}


is awake. The child can then return to sleep. A sleep diary should be continued throughout treatment to monitor progress. The scheduled awakenings should be completed every night for the first week of treatment.

\section{Treatment Fading and Termination}

If no episodes have occurred during the first week of treatment, treatment fading can begin. Treatment fading involves slowly reducing the number of scheduled awakenings each week by skipping one night during the week (e.g., complete six out of seven awakenings), and skipping additional nights each subsequent week (one per week, e.g., five out of seven awakenings, then four out of seven). This should be continued until the scheduled awakenings have been completely phased out $[6,16]$.

If the child continues to have any parasomnia episodes during the initial treatment week, nightly scheduled awakenings should be continued for subsequent weeks until the child goes an entire week without any episodes before initiating treatment fading. Similarly, if parasomnia episodes return during the process of treatment fading, parents should resume nightly scheduled awakenings until the child again goes an entire week without episodes and fading can continue. Treatment is completed once scheduled awakenings have been completely discontinued. If parasomnia events recur in the future, the same procedure can be utilized.

\section{Treatment Challenges}

Parents may find scheduled awakenings difficult to implement consistently, in part due to the difficulty of staying up or waking up in order to arouse their child [6]. Indeed, one study found that all caregivers ended the intervention prematurely, presumably due to burden [6]. However, the recommended length of treatment varies among studies, and there is no absolute recommendation for treatment duration. Educating parents on the treatment challenges and advising them to view treatment in weekly increments may help to minimize this burden. Weekly consultation between the family and the clinician is recommended in order to collaboratively make decisions on modifying or terminating treatment on a week by week basis [16]. On the whole, however, parents report high satisfaction with the intervention and treatment improvements [6].

Timing of the scheduled awakenings may be challenging. There may be times when the awakening triggers a parasomnia episode, or the child becomes fully alert and remains awake for a long duration. If this continues for several consecutive nights, the timing of the scheduled awakening should be advanced (i.e., moved earlier) by $15 \mathrm{~min}$. Similarly, if a child has an episode before the scheduled awakening time, the awakening time should be advanced. Delaying the scheduled awakening may be needed if the episodes do not resolve but begin occurring at a later time during the night after the treatment has been implemented [16].

\section{Alternative Treatments}

\section{Technological Advancements}

The Lully Sleep Guardian (Lully, San Francisco, CA) is a device recently on the market that utilizes timed vibrations to improve sleep terrors. The Sleep Guardian device is approximately the size of a laptop computer and is placed under the child's mattress [17]. The device purports to use an algorithm to identify the appropriate time to partially awaken the child using vibrations, reportedly to prevent night terrors without fully disrupting sleep, and is controlled via an application on parents' mobile devices. The Lully Sleep Guardian appears to utilize the same principles as scheduled awakenings but eliminates the need for parents to wake the child. While no published data are currently available, an ongoing clinical trial is in progress to test the device's effectiveness in children ages 2-12 years old.

\section{Medication Management}

While full consideration of the rationale and evidence base for medication therapy for NREM parasomnias is not within the scope of the current article, medications are at times used to treat parasomnias. Ideally, medication management should be reserved for cases of persistent, very severe, and frequent parasomnias that present a risk of injury to self or others [5]. Benzodiazepines and tricyclic antidepressants given approximately $1 \mathrm{~h}$ before bedtime have been reportedly used with success due to their suppressing effect on slow-wave sleep $[5,15,18]$. Dosage should be titrated to avoid side effects of daytime sedation.

\section{Mechanism for Treatment Success}

The mechanism by which scheduled awakenings helps to resolve parasomnias is unknown; however, there are several hypotheses. One hypothesis is that the awakenings reorganize the child's sleep patterns so the underlying electrophysiology of the partial arousal is either prevented or eliminated $[6,16$, 19]. However, this does not explain why the events do not return once treatment is discontinued. One theory is that children may become conditioned to spontaneously self-arouse at the time of the scheduled awakenings, thereby self-continuing the intervention independently [16, 20]. Lastly, intervention studies document increases in the total sleep time of patients following scheduled awakening treatment which suggests that 
the efficacy of scheduled awakenings may be due to increased sleep duration and the related reduction in the density of slowwave sleep [16].

\section{Treatment Efficacy}

Research for treatment of parasomnias is limited and current evidence primarily comes from case studies or small intervention studies. No randomized clinical trials of scheduled awakenings have been conducted to this date. However, the limited evidence that is available is compelling and consistent. Three studies examined scheduled awakenings for sleepwalking, including a multiple-baseline controlled study of three participants [20], and case studies of a 7-year-old and 8-year-old boys [21, 22]. For sleep terrors, Lask [19] treated 19 children ages 5-13 years with scheduled awakenings and found resolution of episodes within 1 week of treatment for all the patients. Sleep terrors later returned for three patients but resolved after treatment was reinstated for an additional week; treatment effects were maintained for all the patients at 1-year post-treatment. Additionally, two separate multiple-baseline studies examined scheduled awakenings for sleep terrors, one in a sample of three healthy boys, and another in three children with autism, showing resolution of episodes following treatment, maintained at 1-year follow-up $[6,23]$. The scheduled awakenings treatment has been classified as a "promising treatment" based on established criteria for evaluation of psychological treatments for sleepwalking and sleep terrors [24]. In sum, scheduled awakenings appear to have utility as a treatment for children with severe and persistent NREM parasomnias, but controlled randomized studies are critically needed.

\section{Conclusions}

NREM parasomnias are common in children but tend to be benign and often remit by late childhood or adolescence. Following a thorough clinical evaluation, the best treatment options can be mutually discussed and agreed upon with parents. Standard treatment recommendations, including safety measures and minimizing triggering factors, are recommended for children with parasomnia episodes that occur infrequently or are of limited severity. Scheduled awakenings is a promising behavioral sleep treatment that can be effective at decreasing or resolving chronic and severe NREM parasomnias in children, but may be burdensome for parents to implement. Careful and ongoing assessment and collaboration between clinicians and caregivers is recommended for optimal outcomes. Given the promising nature of scheduled awakenings yet limited research to date, there is a strong need for additional research including randomized clinical trials to further determine treatment efficacy. Additionally, both epidemiological and treatment research is necessary for sleep-related eating disorder in children.

\section{Compliance with Ethical Standards}

Conflict of Interest Stacey L. Simon declares that she has no conflict of interest. Kelly C. Byars declares that he has no conflict of interest.

Human and Animal Rights and Informed Consent This article does not contain any studies with human or animal subjects performed by any of the authors.

\section{References}

Papers of particular interest, published recently, have been highlighted as:

- Of importance

1. American Academy of Sleep Medicine. International classification of sleep disorders third edition. Darien: American Academy of Sleep Medicine; 2014.

2. Markov D, Jaffe F, Doghramji K. Update on parasomnias: a review for psychiatric practice. Psychiatry (Edgmont). 2006;3(7):69-76.

3. Owens LJ, France KG, Wiggs L. Behavioural and cognitivebehavioural interventions for sleep disorders in infants and children: a review. Sleep Med Rev. 1999;3(4):281-302.

4. Bruni O et al. NREM sleep instability in children with sleep terrors: the role of slow wave activity interruptions. Clin Neurophysiol. 2008;119(5):985-92.

5. Mason 2nd TB, Pack AI. Pediatric parasomnias. Sleep. 2007;30(2): $141-51$.

6. Durand VM, Mindell JA. Behavioral interventions for childhood sleep terrors. Behav Ther. 1999;30(4):705-15.

7. Klackenberg G. Incidence of parasomnias in children in a general population. In: Guilleminault C, editor. Sleep and its disorders. New York: Raven; 1987. p. 99-113.

8. Ozgun N, et al. Insomnia and parasomnia frequency and affecting factors in school children. Pediatr Int. 2016. doi:10.1111 /ped.12954. Utilizing a large sample of Turkish school-age children, this questionnaire-based study described prevalence rates of sleep disorders, including parasomnias.

9. Auger RR. Sleep-related eating disorders. Psychiatry (Edgmont). 2006;3(11):64-70.

10. Winkelman JW, Herzog DB, Fava M. The prevalence of sleeprelated eating disorder in psychiatric and non-psychiatric populations. Psychol Med. 1999;29(6):1461-6.

11. Sadeh A. Cognitive-behavioral treatment for childhood sleep disorders. Clin Psychol Rev. 2005;25(5):612-28.

12. Petit D et al. Childhood sleepwalking and sleep terrors: a longitudinal study of prevalence and familial aggregation. JAMA Pediatr. 2015;169(7):653-8. This paper examined a large prospective longitudinal sample and reported prevalence of childhood sleepwalking and sleep terrors and provided evidence that family history is a predisposing factor for parasomnias.

13. Guilleminault $\mathrm{C}$ et al. Sleepwalking and sleep terrors in prepubertal children: what triggers them? Pediatrics. 2003;111(1):e17-25.

14. Schenck CH, Mahowald MW. On the reported association of psychopathology with sleep terrors in adults. Sleep. 2000;23(4):448-9. 
15. Wills L, Garcia J. Parasomnias: epidemiology and management. CNS Drugs. 2002;16(12):803-10.

16. Byars K. Scheduled awakenings: a behavioral protocol for treating sleepwalking and sleep terrors in children. In: Perlis ML, Aloia M, Kuhn BR, editors. Behavioral treatments for sleep disorders: a comprehensive primer of behavioral sleep medicine treatment protocols. London: Elsevier Inc.; 2011.

17. Hite E. Biodesign fellows develop a device for relieving night terrors. In: Inside Stanford magazine. 2014. p. 3.

18. Roehrs T, Roth T. Drug-related sleep stage changes: functional significance and clinical relevance. Sleep Med Clin. 2010;5(4): 559-70.
19. Lask B. Novel and non-toxic treatment for night terrors. BMJ. 1988;297(6648):592.

20. Frank $\mathrm{NC}$ et al. The use of scheduled awakenings to eliminate childhood sleepwalking. J Pediatr Psychol. 1997;22(3):345-53.

21. Clement PW. Elimination of sleepwalking in a seven-year-old boy. J Consult Clin Psychol. 1970;34(1):22-6.

22. Tobin Jr JD. Treatment of somnambulism with anticipatory awakening. J Pediatr. 1993;122(3):426-7.

23. Durand VM. Treating sleep terrors in children with autism. J Posit Behav Interv. 2002;4(2):66-72.

24. Kuhn BR, Elliott AJ. Treatment efficacy in behavioral pediatric sleep medicine. J Psychosom Res. 2003;54(6):587-97. 